\title{
Performance Evaluation of different Transport layer protocols on the IEEE 802.11 and IEEE 802.15.4 MAC/PHY layers for WSN
}

\author{
Atif Sharif \\ Digital Ecosystems and Business \\ Intelligence Institute \\ Curtin University of Technology, Perth, Curtin University of Technology, Perth, \\ Western Australia \\ atif.sharif@postgrad.curtin.edu.au
}

\author{
A.J.D.Rathnayaka \\ Department of Electronic and \\ Telecommunication Engineering, \\ University of Moratuwa, Sri Lanka \\ ajdr03@ent.mrt.ac.lk
}

\begin{abstract}
Wireless Sensor Networks (WSN) has gathered lot of attention from the research community lately. Among other WSN communication protocols, transport layer protocol plays a significant role in maintaining the node's energy budget. In this context we have carried out extensive testing of various transport protocols using IEEE 802.11, IEEE 802.15.4 MAC/PHY protocol and Ad hoc On-Demand Distance Vector Routing (AODV) routing agent for WSN having multi-hop ad-hoc and WPAN network topology. The main contribution of this paper is to find out the dependency of Transport layer on MAC layer. Simulation results indicate that the underlying $\mathrm{MAC} / \mathrm{PHY}$ layer protocol along with Transport layer protocol plays a vital role in achieving the high throughput, low latency and packet loss rate in WSN. For IEEE 802.11 with RTS/CTS ON high throughput, low packet drop rate and increased end-to-end packet delay is observed. While for IEEE 802.15.4 similar behavior as for IEEE 802.11 (except for UDP) but with improved power efficiency is observed. This has led the foundation for the future development of the proposed cross layered energy efficient transport protocol for multimedia application.
\end{abstract}

\section{Keywords}

Transport Protocol, IEEE 802.11, IEEE 802.15.4, TCP, UDP, Reliability, Congestion Control.

\section{INTRODUCTION}

The idea of low power, low cost, embedded wireless devices capable of gathering useful environmental information has led the development of Wireless Sensor Networks (WSNs)[1-2]. Today WSN technology has gained fundamental importance in a range of application scenarios like military, modern healthcare, environmental monitoring, surveillance etc. Fundamentally energy is considered as the biggest threat to WSN mote design including both the hardware and the communication protocol design. Majorly WSN pays high energy cost in data retransmissions occurred due to path loss, congestion and high bit

Permission to make digital or hard copies of all or part of this work for personal or classroom use is granted without fee provided that copies are not made or distributed for profit or commercial advantage and that copies bear this notice and the full citation on the first page. To copy otherwise, or republish, to post on servers or to redistribute to lists, requires prior specific permission and/or a fee.

MoMM2009, December 14-16, 2009, Kuala Lumpur, Malaysia. Copyright 2009 ACM 978-1-60558-659-5/09/0012...\$10.00. error rate (BER) caused by interference or collisions at the receiving node. Either case has a direct impact on the overall network throughput and end-to-end packet delay. Reliable and high data transfer rate are the main concerns in event-critical applications e.g. Wireless Multimedia Sensor Network (WMSN). So facilitating congestion control and end-to-end reliability mechanism will be the primary objective of transport layer protocol. In WSN, the transport function's reliability component of WSN communication protocol stack handles minimum number of data packets (either scheduled or prioritized) in order to ensure the minimum latency and energy conservation (achieved by minimum retransmissions) under congested network conditions. Also the transport function must ensure the event and query reliability. The WSN network dealing with multimedia information, i.e. WMSN, the effect of congestion is even more pronounced. The nodes near the sink or those who are experiencing large amount of data above their handling capacity (bottleneck nodes) experience congested paths during data transmission that requires high data rate. This condition would result in performance degradation (due to collisions and retransmissions) and rapidly depletes the node's energy. Thus the transport protocol should effectively detect the congestion and try to mitigate it immediately in order to avoid excessive collisions and retransmissions.

We first start discussing the TCP [17] and UDP [18] schemes of the existing wireless network. TCP is a connection oriented, three way handshake schemes which assume segment losses results from congestion. It provides congestion control and end-to-end reliability mechanism but at high energy cost because of data packets retransmissions under strict end-to-end reliability constraint. UDP on the other hand has neither flow control nor congestion control, so no such constraint of reliability at all. Although it is energy efficient but lacks reliability. However, the modified TCP like STCP [10] is better than UDP for WMSN.

New proposals using multi-path transmission have been introduced such as Congestion Detection and Avoidance (CODA) [7] and Multi-flow Real-time Transport Protocol (MRTP) [5]. CODA has considerable delay since it decides on the basis of the status of the intermediate nodes and MRTP does not consider energy efficiency in WMSN. Some other transport protocols have been designed recently for scalar WSNs with the aim of decreasing energy consumption, providing reliability, and controlling congestion. These protocols did not fulfill major factors of QoS, such as high bandwidth and real-time 
communication, which are required for multimedia communication in sensor networks, e.g. RMST [9], RBC [8], and STCP [10] do not support real-time communication while providing reliability. Also, Fusion [11] is another transport protocol that is not compatible with the limited energy sources of video sensor nodes. Also existing work in transport protocol development targets either upstream (source-to-sink) [12, 13] or downstream (sink-to-source) reliability [14, 15, and 9].

In this paper we primarily focus on the role of transport agent for ensuring data delivery while keeping in the constraints of energy cost. We will test this by observing the behaviors of standard transport agents like UDP and TCP including its variants like Tahoe, Reno, NewReno, Vegas, SACK, and Fack etc over various MAC/PHY layer protocols in wireless domain using AODV [21] as a routing agent. This helps in understanding the dependence of transport layer functionality over MAC/PHY layer.

The rest of the paper is organized as follows. Problem definition is introduced in section 2 followed by section 3 where we describe the simulation setup used for observing the proposed protocol behavior. In section 4 we show the simulation results we have observed. The discussion followed by the conclusions will be presented in the section 5 .

\section{PROBLEM DEFINITION}

In wireless sensor network considerable amount of energy is consumed for per packet communication. This high energy cost is due to many factors including packet collisions at the receiving node due to transmissions from other nodes having the common destination, crosstalk: resulting in high bit error rate (BER), congestion, route failure etc. All these factors contribute to high energy cost, increased end-to-end packet delay and lower throughput of the system.

To cut down the overall networks energy budget and likewise to enhance the effective throughput of the system by minimizing the end-to-end packet delay we have to envisage a transport protocol that takes all these effects into account. Indeed the envisaged transport protocol actually can get most of the information from the MAC/PHY layer. So the purpose of the simulation is to evaluate the existing standard transport protocols for wireless scenario. This simulation helps us in better understanding of the above said performance limiting features and would allow us to possibly look for new dimensions in transport protocol designing for WSN.

\section{SIMULATION SETUP AND PERFORMANCE METRICS}

\subsection{Network Plan}

In this section we will describe the network plan which will be used in extensive functionality testing of UDP, TCP and its variant like Tahoe, Reno, NewReno, Vegas, SACK, and Fack etc for WSN. The network topology (homogeneous by nature) we have used is shown in the Fig 1 and is comprised of 9 wireless sensor nodes placed in open/free space environment. Node $0,1,2$ and 3 are considered as source nodes having TCP/TCP variants or UDP as transport agents and File Transfer Protocol (FTP) or Constant Bit Rate (CBR) as traffic sources. The packet size is considered to be $1 \mathrm{~KB}$ in each case and in CBR the data rate is set to $400 \mathrm{Kbps}$ for effective utilization of the bandwidth. The Node 8 acts as a sink while rest of the nodes act simply as relay nodes (only involved in routing). The motive of the test setup is to evaluate the dependence of transport protocol on MAC/PHY layer by measuring the network's throughput, packet loss ratio, end-toend packet delay and energy behavior of nodes by employing the node's energy model.

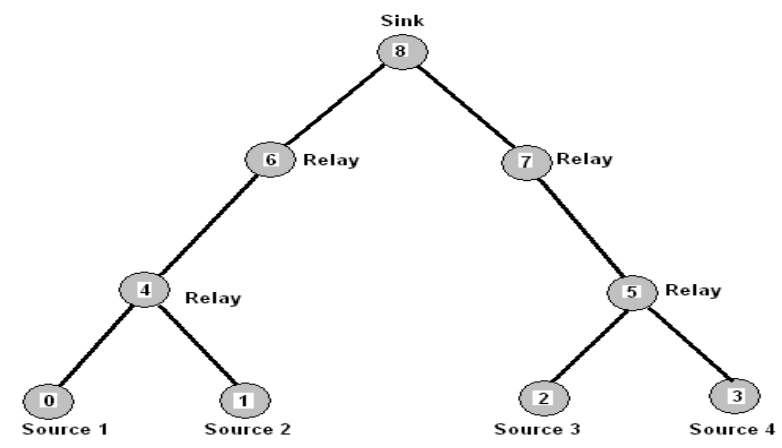

Fig1. The Network topology used for evaluation

\subsection{Radio Propagation Model (RPM)}

The RPM is generally used to predict received packet power, which is entirely decided by the path loss $(L)$, the loss covered by the distance between the transmitter $(t x)$ and receiver $(r x)$. If the received packet power is above than certain threshold limit then it is considered as successfully received packet else the packet is considered to be erroneous and dropped. We used open space scenario for our simulation. In open space the receiving node at a distance 'd' from the transmitter receives signal from a direct path as well as from ground reflections. And for our network we will use the TwoRayGround as radio propagation model. This model considers both the ground reflections and direct path. For this case the received signal power $\mathrm{P}$ at the receiver $(r x)$ is given by $\mathrm{P}_{\mathrm{rx}}$ $[19,20]$

$$
P_{r x}=\frac{P_{t x} \cdot G_{t x} \cdot G_{r x} \cdot h_{t x}{ }^{2} \cdot h_{r x}{ }^{2}}{d_{t x-r x}{ }^{4} \cdot L}
$$

Where $G_{t x} G_{r x}$ is the cumulative gain of the transmitting and receiving antenna, $h_{t x} \& h_{r x}$ are the effective heights of transmitting and receiving antennas, $\mathrm{d}_{\mathrm{tx}-\mathrm{rx}}$ is the distance between transmitter and receiver node and $\mathrm{L}$ is called the path loss.

Before going to find the signal to noise ratio (SNR) threshold at the receiver as we mentioned above we first shortly define the interference region, the region linked to the receiver having radii $\mathrm{d}_{\text {int }}$, in which the receiving node may be interfered with another transmission (interfering transmission power $\mathrm{P}_{\text {int }}$ ) from a hidden node at a distance $\mathrm{d}_{\text {int }}$ from it. So the SNR threshold (SNR_Thres) at the receiver is given by the following equation [20]

$$
S N R_{r e c}=\frac{P_{r x}}{P_{\text {int }}}=\left(\frac{d_{\text {int }}}{d_{t x-r x}}\right)^{4} \geq S N R_{-} \text {Thres }
$$

(for successful reception)

(Eq. 2) 
So to avoid the packet collision at the MAC level due to hidden node (also called interfering node) the $\mathrm{d}_{\text {int }}[20]$ should be

$$
d_{\mathrm{int}} \geq \sqrt[4]{\mathrm{SNR} \text { Thres }} * d_{t x-r x}
$$

By the proper selection of the $d_{\text {int }}$, hidden nodes appear out of the receiving node's interference region and this minimize the effect of packet collisions at the receiving node due to transmissions from hidden nodes. So in this case the receiving node is insensitive to the hidden node's communication and this would result in a collision free communication. So it is an important point to consider $\mathrm{d}_{\text {int }}$ while network design.

\subsection{MAC Protocol}

We used IEEE 802.11[4] and IEEE 802.15.4 [3] as MAC/PHY layer standards. Both protocols use Carrier Sense Multiple Access with Collision Avoidance (CSMA/CA) for carrier sensing and collision avoidance and operates in $2.4 \mathrm{GHz}$ ISM band. In IEEE 802.11 we also exploit the use of Request to Send frame/Clear to Send frame (RTS/CTS) for avoiding the hidden node problem in ad-hoc network.

Normally we take SNR_Thres $=10$ for quality reception, so from eq3 we will get

$$
\begin{aligned}
& d_{\mathrm{int}} \geq 1.778 * d_{t x-r x} \\
& d_{t x-r x} \geq 0.5624 * d_{\mathrm{int}}
\end{aligned}
$$

Although by making carrier sensing region having radius $d_{c s}>d_{\text {int }}$ we can avoid collision since the interfering node actually have to wait for the carrier for its transmission, which is currently being used by the main transmitting node. However this substantially degrades the overall system throughput. Similarly by setting $\mathrm{d}_{\mathrm{cs}}=$ $\mathrm{d}_{\text {int }}$ avoids the use of RTS and CTS. So we have to compromise in between the two thresholds.

\subsection{Energy Model}

To test the energy efficiency of the transport layer protocols for WSN using IEEE802.11 [4] and IEEE802.15.4 [3] as MAC/PHY layer standards and Ad hoc On-Demand Distance Vector Routing (AODV) as routing agent we employ the energy model in our simulations. Since every node consumes some power during transmission, reception and idle state, this model helps in keeping track of the energy graph of individual node. The purpose is to check the effectiveness of the transport protocol from the energy consumption point of view. Since more retransmissions from a particular node as a result of packet collisions due to blind node or poor SNR due to bad channel quality would result in rapid depletion of node's energy.

\subsection{Performance metrics}

The performance of various transport protocols is evaluated against the following metrics

1. Average end-to-end throughput: It is defined as the ratio of data send by all the potential sources to the data received by the sink by taking into account the channel bandwidth.

2. Average end-to-end packet delay: It is defined as the total time a packet would take from the source to sink (end-to-end). This includes all the possible delays as a result of queuing, MAC layer retransmissions, propagation delays and transfer time.

3. Percentage Average data loss: It is defined as ratio of the data loss (difference of send and received data) to the send data multiplied by 100 . The main contributors of data loss are collisions at the receiving end in the presence of blind nodes, congestion and link failure due to node's energy depletion. Here we also take into account the effect of collisions due to blind nodes.

4. Average Power consumed: It is defined as the average power, in Watts, consumed by the source, relay and sink nodes during the data communication over wireless channel including the possible retransmissions at the PHY/MAC level.

Using equations 1-5 we will now design a wireless network comprised of 9 nodes and will then test the functionality of UDP, TCP and its variant like Tahoe, Reno, NewReno, Vegas, SACK, and Fack against the performance metrics as mentioned in section 3.5.

\section{SIMULATION RESULTS}

In this simulation we have used Network Simulator NS-2 [16] and we consider that the entire multi-hop network is comprised of 9 nodes as an ad-hoc network for the case of IEEE 802.11 and multi hop WPAN for the case of IEEE 802.15.4 (Sink (node 8) act as a PAN coordinator). For these wireless setups we performed the extensive analysis of total throughput, end-to-end packet delay and the average power consumed by the source, relay and sink nodes. In this simulation setup we focused more on results from practical perspective and for this we have used the following parameters listed in the Table 1.

Table1. Wireless Sensor Network Parameters

\begin{tabular}{|l|c|}
\hline \multicolumn{1}{|c|}{ Parameter } & \multicolumn{1}{c|}{ Values } \\
\hline Frequency & TwoRayGround \\
\hline Propagation model & $\begin{array}{l}\text { UDP, } \\
\text { TAHOE, TCP-SACK } \\
\text { VENG, NEW-RENO, } \\
\text { FACK }\end{array}$ \\
\hline Transport Protocols & $\begin{array}{c}\text { IEEE802.11 and } \\
\text { IEEE802.15.4 }\end{array}$ \\
\hline MAC & $\begin{array}{c}1.296 \mathrm{e}-10 \mathrm{~W} \\
\text { RX Threshold }\end{array}$ \\
\hline IEEE802.11 (node separation 250m) & AODV \\
\hline IEEE802.15.4(node separation 50m) & 200 packets \\
\hline Routing agent & Yes \\
\hline Ifqlen (Queue length at MAC level) & 10 \\
\hline Energy Model & $1.1664 \mathrm{e}-10 \mathrm{~W}$ \\
\hline CP Threshold & $3.357594 \mathrm{e}-9 \mathrm{~W}$ \\
\hline CS Threshold & $100 \mathrm{~W}$ \\
\hline IEEE802.11 & $712 \mathrm{e}-6 \mathrm{~W}$ \\
\hline IEEE802.15.4 & $35.28 \mathrm{e}-3 \mathrm{~W}$ \\
\hline Node initial power & $31.32 \mathrm{e}-3 \mathrm{~W}$ \\
\hline Node idle power consumption & \\
\hline Node tx power consumption & \\
\hline
\end{tabular}




\begin{abstract}
Node sleep power consumption
$0.001 \mathrm{~W}$

The values for RX Threshold and CS Threshold are calculated by using the idea presented in eqs (1-5). Also in our case we are actually considering the amount of power consumed by nodes (MICA motes) during their communication, idle and sleep modes by enabling the Energy Model parameter. Also we are considering the case of multimedia application with a packet size of $1 \mathrm{~KB}$ and sending rate is $400 \mathrm{Kbps}$ (for each source) for UDP where as for TCP it grows based on its congestion window. The simulation is performed 5 times and the results mentioned here are averaged for each particular case.
\end{abstract}

\subsection{IEEE 802.11}

For the case of IEEE 802.11 we performed simulation for two cases

\section{- $\quad \mathrm{RTS} / \mathrm{CTS}$ ON and}

\section{- $\quad$ RTS/CTS OFF}

\subsubsection{Throughput Vs Reliability}

From Fig 1 and Fig 2 it is obvious that with RTS/CTS ON the data loss rate (due to collisions) reduces and throughput increases which results in power efficiency by eliminating the need of packet retransmission at the MAC/PHY level.

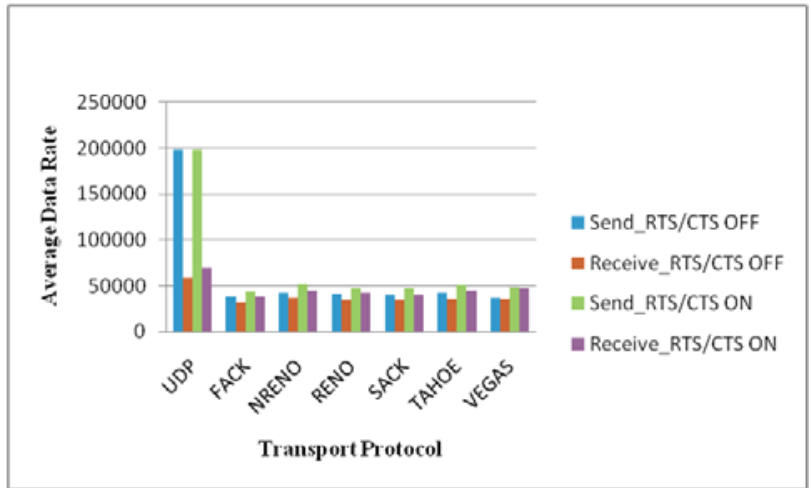

Fig 1. Average Data Send/Received (Bytes) using IEEE 802.11 with RTS/CTS OFF/ON

Also among various transport protocols UDP has showed its poor reliability and throughput. Its throughput is marked to about $29.72 \%$ and $35.18 \%$ for RTS/CTS OFF and RTS/CTS ON cases and is significantly lower than TCP and its variants. Where as in case of TCP, throughput is around $86 \%$, only TCP-Vegas showed better result in case of data reliability having $5.6623 \%$ and $3.040613 \%$ data loss respectively while others have $12-16 \%$. But with respect to the available bandwidth of $2 \mathrm{Mbps}$ (effectively 1.7 Mbps [6]) the bandwidth utilization factor of TCP is poorer as compared to UDP. Also in comparison to the received number of bytes for the two cases due to packet collision, RTS/CTS OFF, at MAC level about $5.46 \%$ of the sent bytes has been lost in case of UDP while for the rest of the cases its averages around $5.5 \mathrm{Kbytes}$ $(3-20 \%)$ which is also quite significant and is not tolerable for event driven applications and also for multimedia sensor applications where data reliability is of great concern. Fig 3 depicts the average power consumed by the source, relay and sink node during the communication.

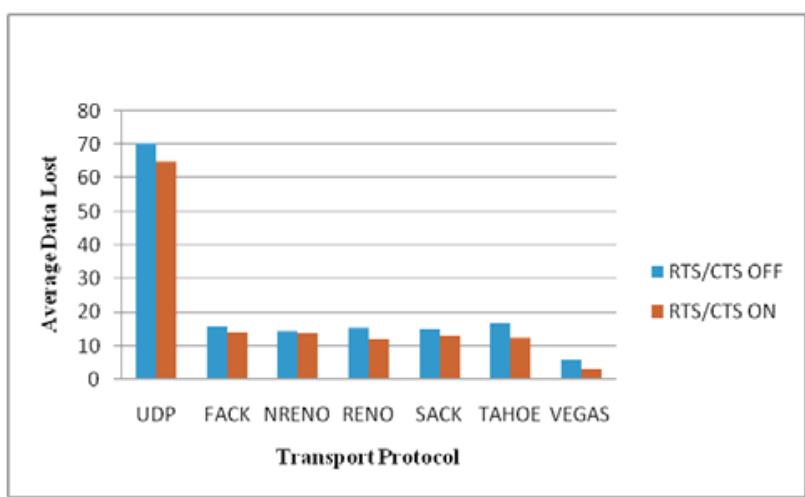

Fig 2. a) Data Reliability Analysis using IEEE 802.11 with RTS/CTS OFF/ON

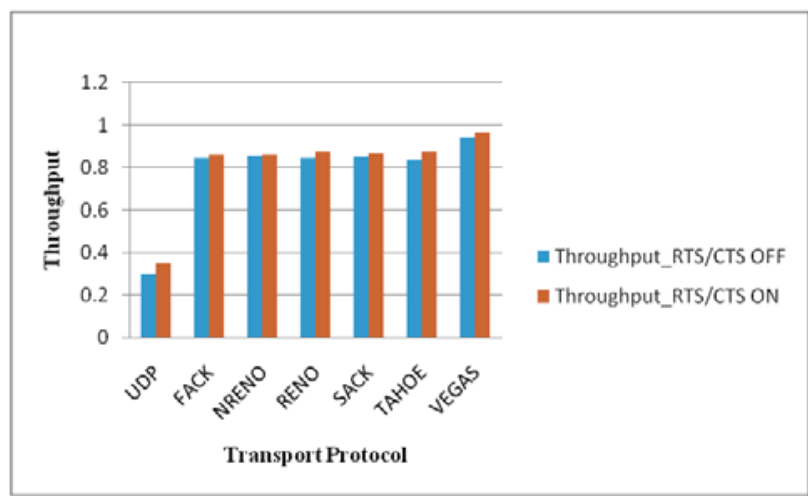

Fig 2. b) Throughput (ratio) Analysis using IEEE 802.11 with RTS/CTS OFF/ON

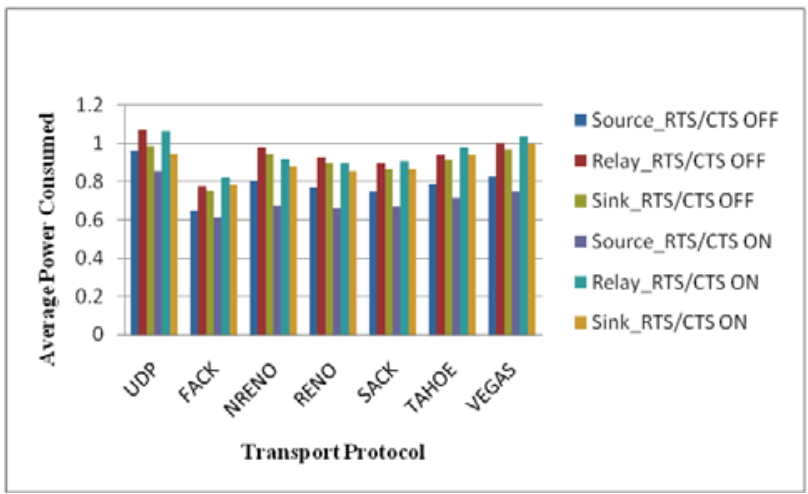

Fig 3. Average Power (Watts) Consumed using IEEE 802.11 with RTS/CTSOFF/ON

From this it would be obvious that with RTS/CTS ON the energy consumption graph of the respective nodes also drops since because of RTS/CTS ON the collisions at receiving side due to blind nodes has been dropped down in comparison to RTS/CTS OFF case where nodes on the average consume 1-3\% higher energy because of retransmissions. And this $1-3 \%$ difference is quite significant especially when we talk about the longevity of such networks. 


\subsubsection{End-to-End Packet Delay}

As we are concerned with multimedia traffic so we can see from Fig4 that with high data rate the data is queued at the MAC level due to congestion and MAC level retransmissions and this queued delay is more dominant in node's average end-to-end packet delay behavior. Also with the inclusion of RTS/CTS ON the sources actually have to wait for the carrier sense before actually start transmission. And for that reason we have seen that with RTS/CTS ON the average end-to-end packet delay has been increased. UDP amongst all again showed poor results in both cases because in case of UDP the CBR traffic source kept sending data packets of size $1 \mathrm{~KB}$ at a rate of $400 \mathrm{Kbps}$. Where as TCP on the other hand has an average end-to-end delay of more than $120 \mathrm{msec}$ and is still did not meet the objectives of multimedia traffic (for voice $\leq 120 \mathrm{msec}$ ).

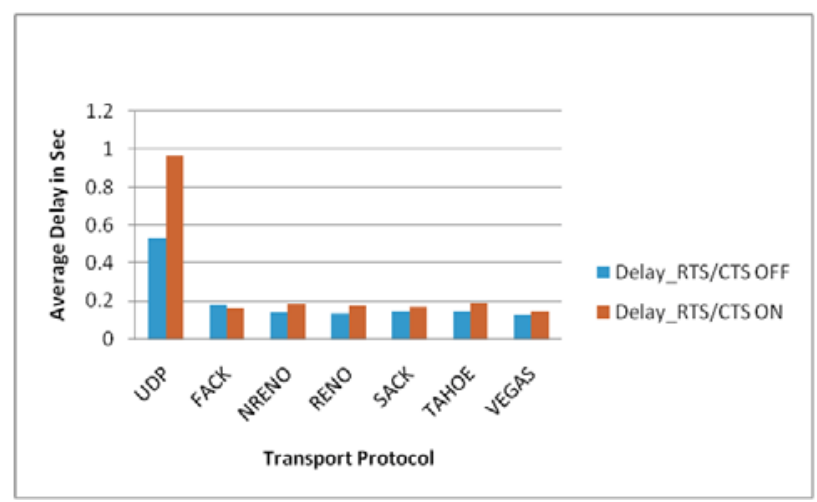

Fig 4. Average End-to-End Packet Delay Analysis using IEEE 802.11 with RTS/CTS OFF/ON

\subsection{IEEE 802.15.4}

For IEEE 802.15.4 we evaluated the transport protocols for multihop WPAN targeting multimedia applications with RTS/CTS ON. The evaluation is made against the performance metrics discussed in section 3.5.

\subsubsection{Throughput Vs Reliability}

From Fig 5a and Fig 6 it is obvious that Tahoe and Reno performed well in comparison to UDP and other TCP variants having lowest percentage data loss of $18.878 \%$ and $19.1011 \%$ or having high throughput ratio.

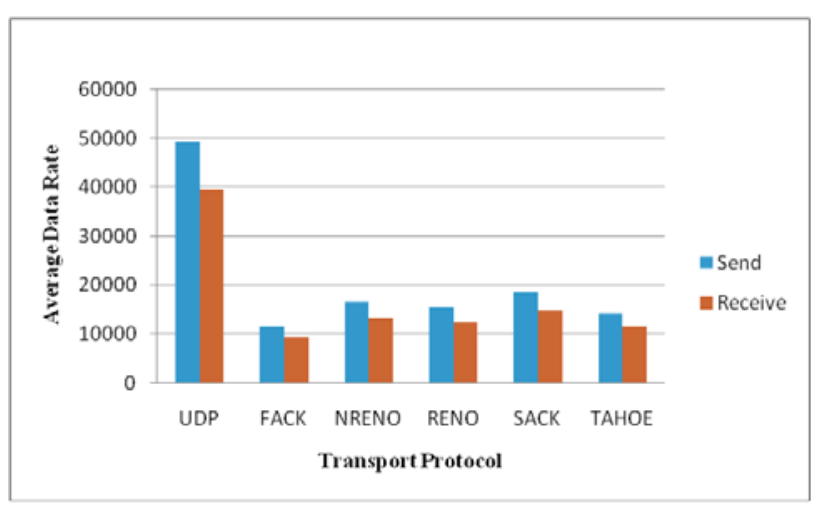

Fig 5. a) Average Data Send/Received (Bytes) using IEEE802.15.4
The UDP has a percentage data loss of $19.54 \%$ which is far better in comparison to the IEEE802.11 case where the percentage data loss was $65-70 \%$.

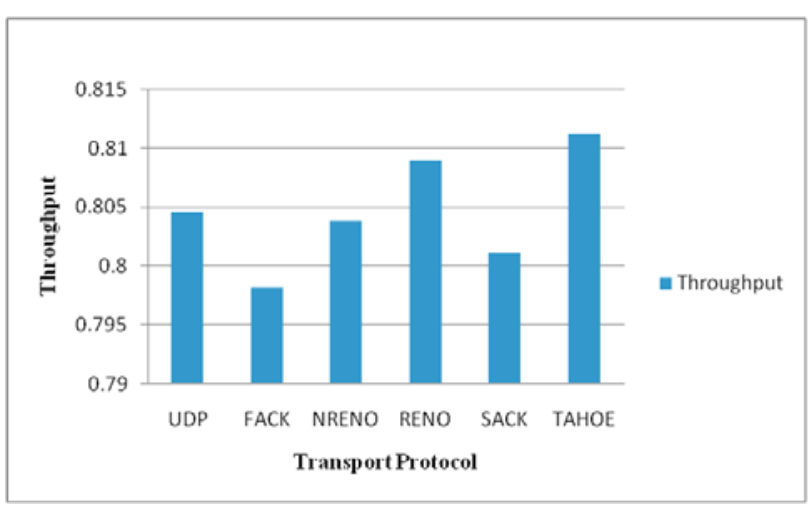

Fig 5. b) Throughput (ratio) Analysis using IEEE802.15.4

From Fig $5 b$ the average throughput of UDP and TCP variants is in the range of $80 \%$. Also for the UDP over IEEE 802.15.4, the throughput result seems promising in comparison with a case of IEEE 802.11 where it was in the range of $29-35 \%$.

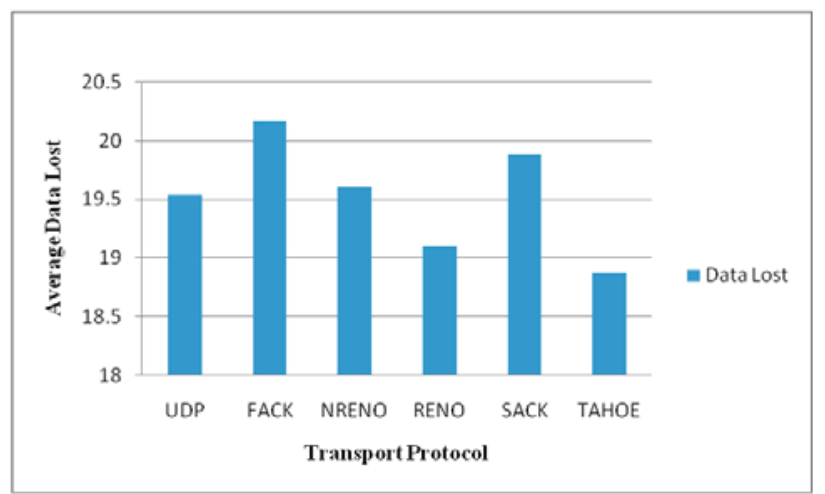

Fig 6. Data Reliability Analysis using IEEE 802.15.4

As we can see from Fig 7 that the average power consumed by the source, relay and sink nodes in WPAN configuration of IEEE 802.15.4 consumes less than half power unit ( 0.5 Watt).

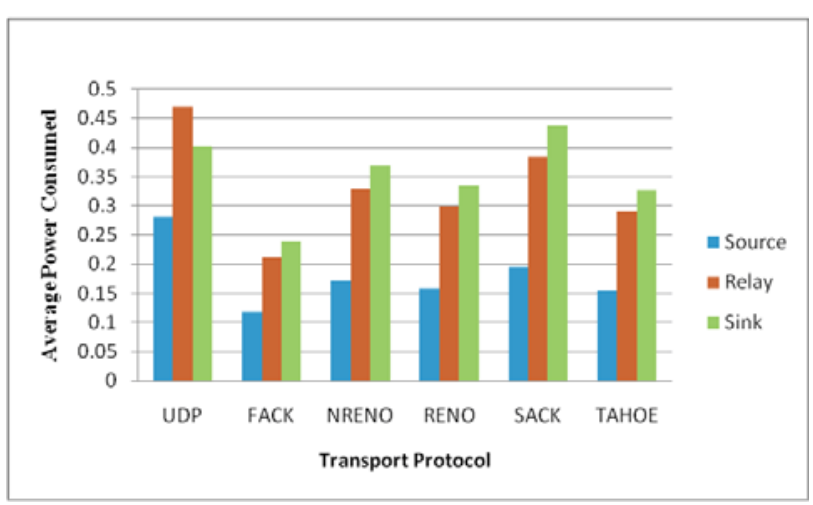

Fig 7. Average Power (Watts) Consumed using IEEE 802.15.4

Although in IEEE802.15.4 configuration the source node sending rate is $250 \mathrm{Kbps}$ (less than the rate (2Mbps) set by IEEE802.11) but still the ratio of successfully received data rate for IEEE 
802.15.4 is better as compared to IEEE 802.11, and this ratio entirely defines why the nodes having IEEE802.15.4 consumed less power in comparison to the case of IEEE 802.11.

\subsubsection{End-to-End Packet Delay}

From Fig 8 we can see that the except SACK, NRENO and TAHOE rest all have an end-to-end packet delay of more than $200 \mathrm{msec}$ which is again not suitable for wireless multimedia and event driven applications.

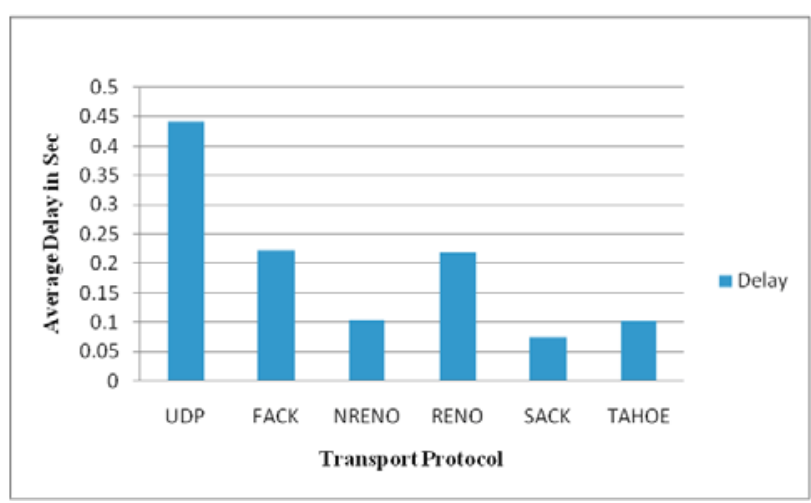

Fig 8. Average End-to-End Packet Delay Analysis using IEEE802.15.4

\section{DISCUSSION AND CONCLUSION}

In this paper we tried to map the impact of MAC/PHY layer protocol on transport protocol functionality, for which we had taken a case of multihop wireless ad-hoc and WPAN for IEEE802.11 and IEEE802.15.4. We actually imposed every possible physical constraint to these networks like the sense range, interference range, bandwidth etc. We also investigated the power consumption of independent nodes in these networks just to graph the power efficiency of these transport protocols. Following conclusions have been drawn from the above simulations

\subsection{IEEE802.11}

Following are the conclusions drawn for the case of IEEE802.11

- With RTS/CTS ON average throughput is increased

- With RTS/CTS ON average packet drop rate is reduced.

- With RTS/CTS ON average end-to-end packet delay is increased.

- With RTS/CTS ON average $1-3 \%$ energy efficiency is achieved.

\subsection{IEEE802.15.4}

Following are the conclusions drawn for the case of IEEE802.15.4

- UDP performed well having high throughput and lower average data loss in comparison to IEEE802.11 with RTS/CTS ON/OFF.

- Approximately on an average $2-4 \%$ more data loss was observed in case of TCP variants in comparison to IEEE802.11.
- For UDP average end-to-end packet delay dropped to a value approximately half to the case of IEEE802.11 with RTS/CTS ON.

- Similar improved average end-to-end packet delay behavior was also observed in case of TCP variants.

- Significant reduction in average power consumption is observed in comparison to IEEE802.11.

We have seen from the above results that there seems to be a direct relationship between the PHY/MAC layer and Transport Layer protocols for getting high throughput and minimal end-toend packet delay. For achieving these objectives we have to take following things into account

1. While network planning the transmitting and receiving node's distances should be calculated according to the equations (1-5) to minimize the packet retransmissions as a result of collisions at the receiving node due to hidden node's transmissions. Here packet retransmission not only introduces the delay but also increase the average per packet power required for successful communication.

2. Also to overcome the effects of hidden node the RTS/CTS should be used at the MAC/PHY level.

3. Another factor, although we did not mention or evaluated above, is the idea of how long the packet is in MAC buffer (buffer delay) and the actual packet processing time (the time the MAC layer receives packet from the network layer to the time it successfully transmit the last bit of the packet). This helps in combating against the end-to-end packet delay challenge by the use of estimation theory for packet processing time at intermediate node.

4. Also from the results it is obvious that a considerable amount of data was lost at MAC/PHY layer protocol, which then reduced the reliability of the transport protocol. The data loss mainly occurred either due to congestion and collisions. Since we addressed the issue of hidden node (means no packet collision at the receiving end) the only thing left was the loss due to congestion. Ideally the protocol should control the rate of data flow and if some data has been lost there should exist some rapid data recovery mechanism. So a need of such data recovery mechanism is necessary.

5. The transport layer protocol should also have a clear picture of the wireless channel quality (packet error rate, PER) so that it is aware of the reason of data loss. Again in this case it will be guided by the MAC/PHY layer.

In summary for achieving high throughput and data reliability for a transport protocol we have to relate the functionalities of the two layers (cross-layering). Merging the functionalities of the two layers enables the transport layer protocol to handle the data more efficiently and delivers it to the destination node more reliably with minimal delay. Also in case of data packet lost which results either due to collisions, crosstalk or path failures due to node's energy depletion etc. would invoke fast data recovery mechanism for retrieving the lost data packet with in marginal time limits without affecting the application's QoS. Based on this point we can predict our future research direction. Our next objective is to implement a transport protocol for wireless multimedia applications that guarantee high throughput by having effective congestion control mechanism while simultaneously provide high 
data reliability by ensuring rapid loss recoveries by introducing the concept of distributed memory storage in WSN.

\section{REFERENCES}

[1] I.F. Akyildiz, T. Melodia, and K.R. Chowdhury, "A Survey on wireless multimedia sensor networks," Computer Networks (Elsevier) J., vol. 51, pp. 921-960, 2007.

[2] V. Potdar, A. Sharif, E. Chang, "Wireless Sensor Networks: A Survey," AINA, pp.636-641, 2009 International Conference on Advanced Information Networking and Applications Workshops, 2009

[3] IEEE 802.15 WPANTM Task Group http://ieee802.org/15/index.html, Aug 2008.

[4] IEEE P802.11, Main General Info Page, Aug 2008. www.ieee802.org/11/main.html, Aug 2008.

[5] S. Mao, D. Bushmitch, S. Narayanan, S. S. Panwar, "MRTP: a Multiflow Real time Transport Protocol for ad hoc networks", IEEE 58th Vehicular Technology Conference, 2003. VTC 2003-Fall. 20032629 - 2634 Vol.4

[6] J. Li, C. Blake, D.S.J. DeCouto, H.I. Lee "Capacity of Ad Hoc wireless networks", Intl Conf on Mobile Computing and Networking Proceedings of the 7th annual international conference on Mobile computing and networking Rome, Italy, PP. 61 - 69, 2001

[7] C.Y. Wan, S.B. Eisenman, and A.T. Campbell, "CODA: Congestion detection and avoidance in sensor networks," Proc. ACM SenSys, pp.266-279, Los Angeles, Nov. 2003.

[8] H. Zhang, A. Arora, Y.R. Choi, M. G. Gouda, "Reliable Bursty Convergecast in Wireless Sensor Networks", Proc. ACM Mobihoc, PP. 266-276, 2005

[9] F. Stann and J. Heideman, "RMST: Reliable Data Transport in Sensor Networks", In Proc. of the First IEEE Int. Workshop on Sensor Network Protocols and Applications, pages 102-113, Anchorage, USA, May 2003.
[10] Y.G. Iyer, S. Gandham, S. Venkatesan, "STCP: a Generic Transport Layer Protocol for Wireless Sensor Networks", in: Proc. of IEEE Intl. Conf. on Computer Communications and Networks (ICCCN), San Diego, CA, USA, PP. 449-454, 2005.

[11] B. Hull, K. Jamieson, H. Balakrishnan, "Mitigating Congestion in Wireless Sensor Networks", In Proceedings of the ACM Conference on Embedded Networked Sensor Systems (SenSys), PP. 134-147, 2004.

[12] S.-J. Park, R. Vedantham, R. Sivakumar, and I. F. Akyildiz, "A scalable approach for reliable downstream data delivery in wireless sensor networks," in Proc. ACM MobiHoc, Japan, PP. 78-89, May 2004.

[13] C. Y. Wan, A. T. Campbell, and L. Krishnamurthy. PSFQ: A Reliable Transport Protocol for Wireless Sensor Networks. In Proc. of ACM Int. Workshop on WSN and Applications, pages 1-11, Georgia, USA, 2002

[14] T. He, J.A. Stankovic, C. Lu, and T. Abdelzaher, "SPEED: A Stateless Protocol for Real-Time Communication in Sensor Networks", In Proc. of ICDCS, Providence, Rhode Island, USA, PP. 46, 2003.

[15] Y. Sankarasubramaniam, O.B. Akan, and I.F. Akyildiz. ESRT: Event-to-Sink Reliable Transport in Wireless Sensor Networks. In Proc. of ACM MobiHoc, pages 177-188, Annapolis, Maryland, USA, June 2003.

[16] The Network Simulator - NS-2, http://www.isi.edu/nsnam/ns/index.html, July 2008.

[17] TCP, http://www.ibiblio.org/pub/docs/rfc/rfc793.txt, Aug 2008.

[18] UDP, http://tools.ietf.org/html/rfc768, Aug 2008.

[19] Li Layuan, Li Chunlin, Yaun Peiyan, "Performance evaluation and simulations of routing protocols in ad hoc networks", Computer Comm 30(8): 1890-1898 (2007)

[20] T.S. Rappaport, "Wireless Communications: Principles and Practice”, Prentice Hall, Upper Saddle River, NJ (1995).

[21] C.E. Perkins, E.B. Royer and S. Das, "Ad hoc On-Demand Distance Vector (AODV) Routing”, RFC 3561, July 2003. 